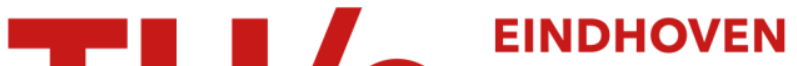 \\ UNIVERSITY OF \\ TECHNOLOGY
}

\section{A formal approach to the automated labeling of groups of features}

Citation for published version (APA):

Reimer, A., Goethem, van, A. I., Rylov, M., Kreveld, van, M. J., \& Speckmann, B. (2015). A formal approach to the automated labeling of groups of features. Cartography and Geographic Information Science, 42(4), 333-344. https://doi.org/10.1080/15230406.2015.1053986

DOI:

10.1080/15230406.2015.1053986

Document status and date:

Published: 01/01/2015

Document Version:

Accepted manuscript including changes made at the peer-review stage

Please check the document version of this publication:

- A submitted manuscript is the version of the article upon submission and before peer-review. There can be important differences between the submitted version and the official published version of record. People interested in the research are advised to contact the author for the final version of the publication, or visit the $\mathrm{DOI}$ to the publisher's website.

- The final author version and the galley proof are versions of the publication after peer review.

- The final published version features the final layout of the paper including the volume, issue and page numbers.

Link to publication

\section{General rights}

Copyright and moral rights for the publications made accessible in the public portal are retained by the authors and/or other copyright owners and it is a condition of accessing publications that users recognise and abide by the legal requirements associated with these rights.

- Users may download and print one copy of any publication from the public portal for the purpose of private study or research.

- You may not further distribute the material or use it for any profit-making activity or commercial gain

- You may freely distribute the URL identifying the publication in the public portal.

If the publication is distributed under the terms of Article 25fa of the Dutch Copyright Act, indicated by the "Taverne" license above, please follow below link for the End User Agreement:

www.tue.nl/taverne

Take down policy

If you believe that this document breaches copyright please contact us at:

openaccess@tue.nl

providing details and we will investigate your claim. 


\title{
A Formal Approach to the Automated Labelling of Groups of Features
}

\author{
Arthur van Goethem*, Marc van Kreveld**, Andreas Reimer ${ }^{\star \star *}$, \\ Maxim Rylov***, Bettina Speckmann* \\ * TU Eindhoven \\ ** Utrecht University \\ **** Heidelberg University
}

\begin{abstract}
A variety of recurring geographic entities form collections of point, line or area features. Examples are groups of islands (Archipelago), relief features in deserts, periglacial lakes or geomorphological forms, such as drumlins and sinkholes. All these groups of features might be best identified with a single label. Surprisingly, the (automated) labelling of groups of features has received little attention so far. We investigate the labelling of feature groups by comparing manually generated labels to a set of formally defined, algorithmically optimal placements. By taking this systematic approach, we hope to detect if, and which, formal measures are-possibly subconsciously-used by cartographers. Furthermore, the framework allows us to define a series of precise algorithmic questions which can readily be investigated in future work.
\end{abstract}

Keywords: Automated Cartography, Labelling, Groups of Features

\section{Introduction}

The ability of a map to convey spatial information is directly related to the quality of its annotation. A clear association between names and their features has a direct influence on the map reading performance (Noyes 1980, Lloyd 1997). Traditionally, the labelling of maps has been a manual process performed by trained cartographers. Map labeling is a labor intensive and time-consuming task which takes a significant part of total map production time. Hence automated cartography, and more generally geographic information systems, have tried to automate the labelling process. Starting with the work by Yoeli (1972), there have been numerous and various attempts (Kern \& Brewer 2008, Wolff \& Strijk 2009) to automate well-established cartographic design techniques (Imhof 1962, Wood 2000, Brewer 2005) for labelling point (settlements, mountain peaks, ...), linear (rivers, boundaries, 
roads, ...) and areal features (countries, islands, lakes, woods, ...). Each design technique introduces its own requirements and challenges. The most complex and challenging task is the labeling of areal features. Areal features allow a high degree of freedom for labeling, due to the great variety of possible shapes to be labeled and the rich and diverse set of cartographic guidelines. For example, the label can be placed inside the area, either horizontally or using a curved label. If the feature is too small for the entire label or contains other important map elements, the label may also be placed outside the area. There is, however, one uniting aspect in all these design techniques: a label should always conform to the shape and the extent of the feature.

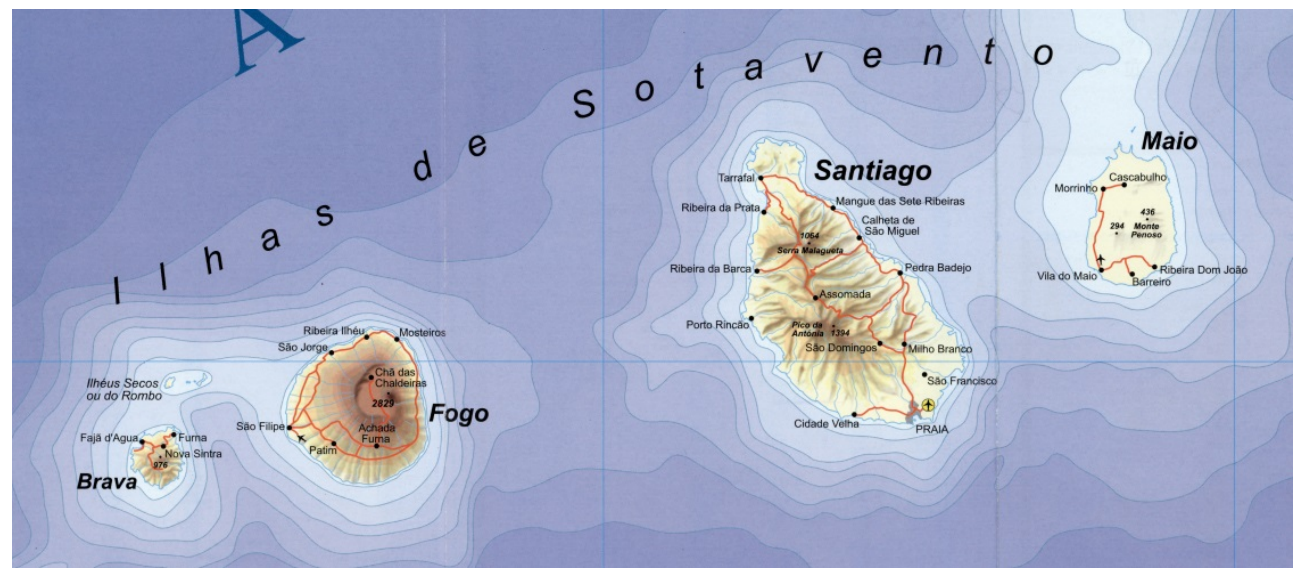

Figure 1. Island group label from the Bézier curve type, shape-aligned orientation, positioned alongside the shape of the group and without any overprinting.

Some geographic features like archipelagos, forest patches, or sand hills can be grouped into a cluster or a chain having a common name (see Figure 1). These feature groups should usually be labelled differently from single areal features. Surprisingly the (automated) labelling of feature groups has received little attention so far. This is at least partially caused by the lack of cartographic principles that can be found in the literature on this subject. Though extensive guidelines for map labelling have been given in seminal works by Imhof (1962), Wood (2000) and Brewer (2005), information on the labelling of feature groups is surprisingly sparse. Wood (2000) suggests that, to label island groups, "...the group name should be on a curve following the general shape of the group or positioned so that the name balances the overall shape of the group." No further guidelines for the labelling of feature groups are known.

Contribution. We investigate the labelling of feature groups by comparing manually generated labels to a set of formally defined, algorithmically op- 
timal placements. After surveying related work (Section 2), we provide an overview of the possible strategies for labelling feature groups as can be observed in manually labelled maps (Section 3). We then (Section 4) define a framework of feasible labeling algorithms. Finally (Section 5) we compare existing, manual labels, replicated from printed atlases, to a subset of the different optimal algorithmic solutions. By taking this systematic approach, we hope to detect if, and which, formal measures are-possibly subconsciously-used by cartographers. To the best of our knowledge this is the first attempt to analyze the techniques used by skilled cartographers in a formal quantitative way. Furthermore, the framework allows us to define precise algorithmic questions pertaining to the automated labeling of feature groups which can readily be investigated in future algorithmic work.

\section{Related work}

Over centuries, cartography as a science and art has developed and collected a wealth of postulates and design techniques for producing clear and legible map labelling (Imhof 1962, Phillips et al. 1977, Wood 2000, Brewer 2005). Almost all existing labelling algorithms (Wolff \& Strijk 2009) heavily rely on cartographic guidelines, such as the ones compiled by Imhof (1962). The task of automated label placement is commonly divided into three subtasks, namely candidate-position generation, position evaluation (van Dijk et al. 2002) and position selection (Christensen et al. 1995). For a detailed explanation of these sub-tasks we refer to Edmondson et al. (1996). Only the sub-tasks of candidate-position generation and evaluation need to use cartographic guidelines.

Many of the techniques used in manual labelling were automated over the last four decades. Algorithms for point labelling were developed in a sliding model by van Kreveld et al. (1999) or in a comprehensive model by Rylov and Reimer (2014a). The problem of labelling linear features was addressed amongst others in Barrault and Lecordix (1995), Edmondson et al. (1996) and Chirié (2000). Van Roessel (1989), Barrault (2001), and Rylov \& Reimer (2014b) proposed methods for labelling areal features. The problem of labelling feature groups has also received some attention. Specifically, van Kreveld and Schlechter (2005) developed an efficient algorithm to label a group of islands. Their algorithm tries to place a horizontal label in the position that "minimizes the maximum distance from the label to each island of the group."

However, there are still several interesting and important design techniques that have not been automated yet. Table 1 shows that point and linear features are most frequently studied, both in the cartographic literature and 


\begin{tabular}{|l|l|l|}
\hline Types of designations & Guidelines & Algorithms \\
\hline Point features & ++ & ++ \\
\hline Linear features & & \\
\hline Centered & ++ & + \\
\hline Alongside & ++ & + \\
\hline Areal features & & \\
\hline Horizontally inside & ++ & + \\
\hline Horizontally outside & ++ & + \\
\hline Straight line inside & ++ & - \\
\hline Curved inside & ++ & + \\
\hline Curved outside & + & - \\
\hline Group of areas & & \\
\hline Horizontal (through) & - & + \\
\hline Straight line (through) & - & - \\
\hline Curved line (through) & - & - \\
\hline Straight line (alongside) & - & - \\
\hline Curved line (alongside) & + & - \\
\hline
\end{tabular}

Table 1. A summary of cartographic guidelines and existing algorithms.

in algorithm design. There are also satisfactory cartographic guidelines for the labelling of areal features, but not many algorithmic solutions as of yet. There are only very few rather unspecific guidelines for groups of areas and-probably as a direct result- hardly any efficient and effective algorithms.

\section{Cartographic Design of Feature Labelling}

Bertin (1967) noted that the cartographic representation of groups of features across scales is one of the most difficult and error-prone tasks within cartography. As he shows in his example of a group of lakes, the problems of emphasis and visual grouping become multidimensional: shape, orientation, Gestalt properties, and non-geometric attributes all compete for the attention of the mapmaker. This has also been recognized as a hard problem in automated generalization (Steiniger \& Weibel 2007).

As with all classifications, grouping geometric objects always keeps some elements of arbitrariness. This puts more pressure on the label: The string "Liverpool", for example, should identify a single point feature on the map representing the town and nothing else. "Spitsbergen", by contrast, should have a clear relationship to all parts of the Archipelago. Following Imhof (1962), the fundamental function of any label is disambiguation. This func- 

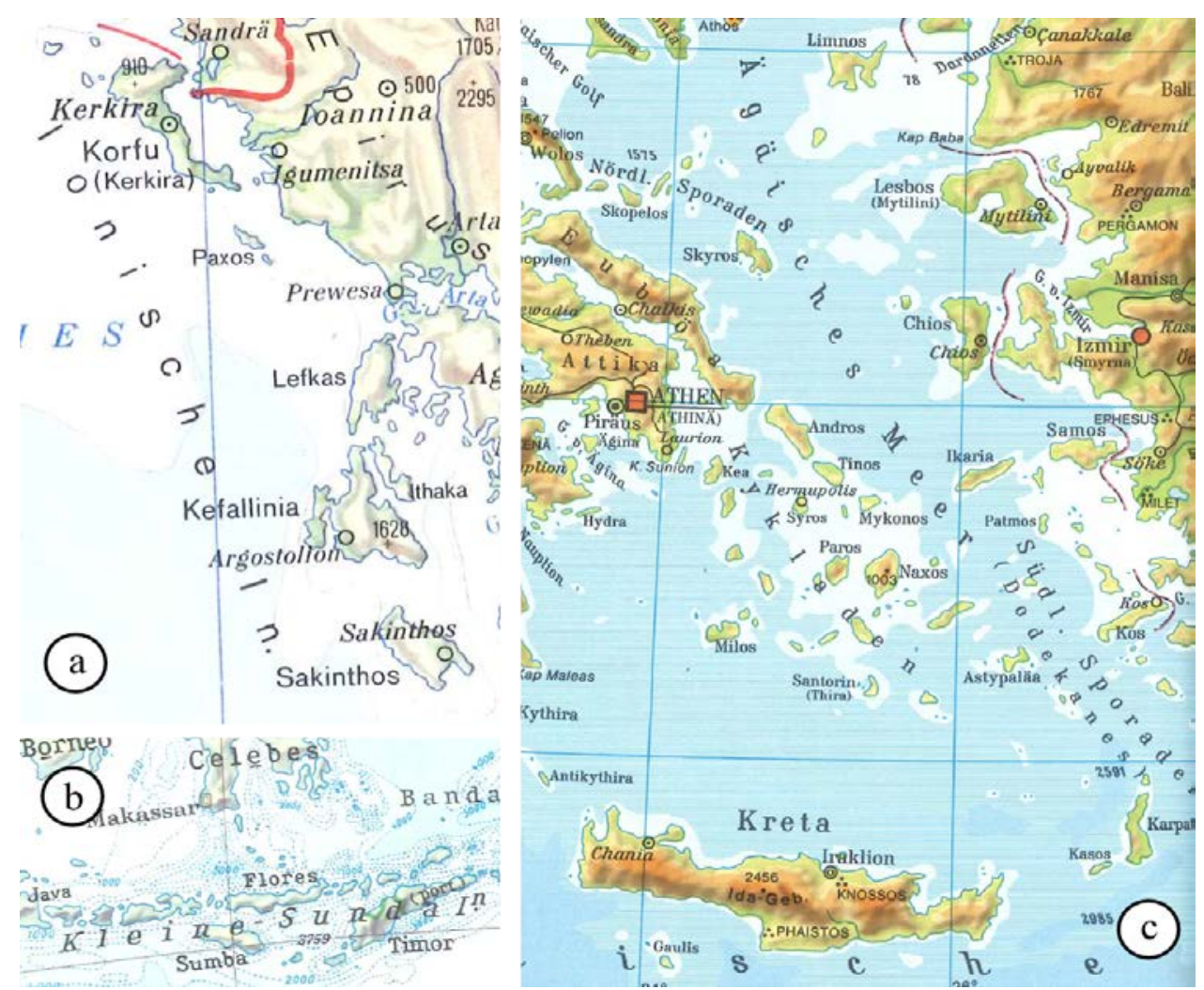

Figure 2. Different placement strategies: (a) Ionische In. Bézier curve, shapeconnecting (b) Kleine Sunda In. Circular Arc, grid-aligned, through the Archipelago, and (c) Nördl. Sporaden circular arc, shape-aligned, connecting; Kykladen circular arc, shape-aligned, through.

tion is naturally harder to achieve for object groups, which are potentially close to other object groups, than for the simple object-label pairs in point labeling. A single label might even be all that ties the objects together visually. Group labels hence have a constructive function in addition to the de scriptive function that all labels carry.

Manually labelled maps show a much greater variety of placements for group labels than for point labels (Figure 2 and 3). Instead of a clear hierarchy of 6-8 preferred positions, we find curved labels, axis-aligned straight lines, rotated straight lines, who all might or might not overlap the constituent features, placed outside or within the group, etc. This greater variety is due to the higher degrees of freedom for both the arrangement and shape of the feature, as well as the position of the label.

It is often even unclear which placement strategy (each in turn warranting its own algorithm) is required under which circumstances. Many non- 

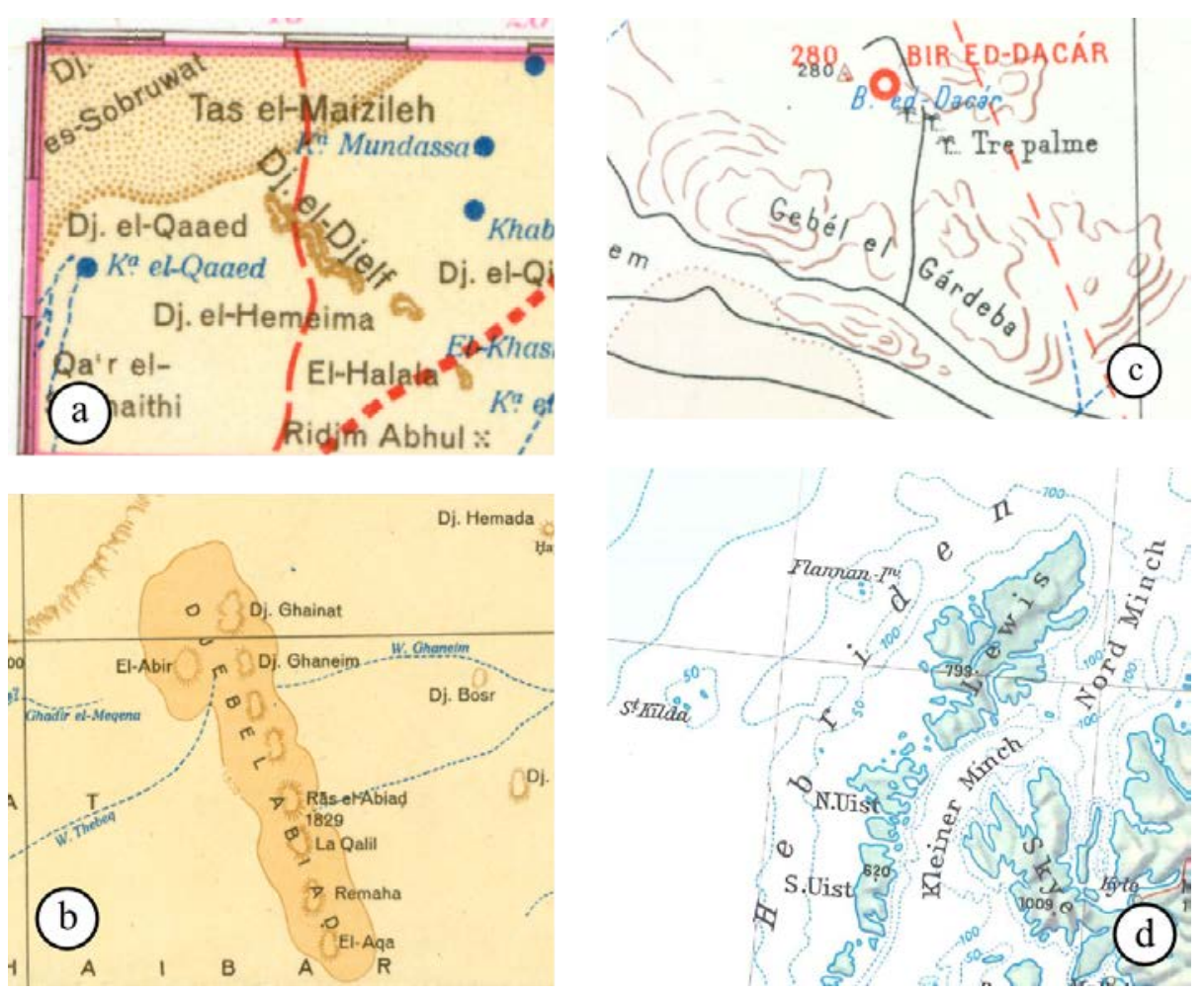

Figure 3. Different placement strategies: (a) Dj. el Djelf Straight-line, shapealigned (b) Straight-line, shape-aligned, through (c) Gebel el Gardeba circular arc, shape-aligned, through for linear elements, and (d) Hebriden circular arc, shape-aligned, alongside; St. Kilda straight line, grid-aligned, accompanying.

geometric determinants appear to play a role. One example are administrative peculiarities, especially regarding international borders, that heavily use the constructive role of the label. But also authoring and reproduction techniques influence placement. Copper engraving and the growing virtuosity some engravers achieved led to toponym densities above 700 toponyms per $(10 \mathrm{~cm})^{2}$ (Figure 4), which could still be reproduced by lithographic processes. Off-set printing paired with manual labelling via rub-off letters or similar techniques lead to lower densities, and thus more degrees of freedom, which were used differently by different cartographers.

Color usage is another non-geometric determinant, especially regarding the visual impact of, and tolerance for, overprinting. Light background colors paired with a full black are much more forgiving of small overprints than on-screen rasterized maps. As a consequence, we distinguish between small overprints which are of no harm in offset-printing and are limited to the 


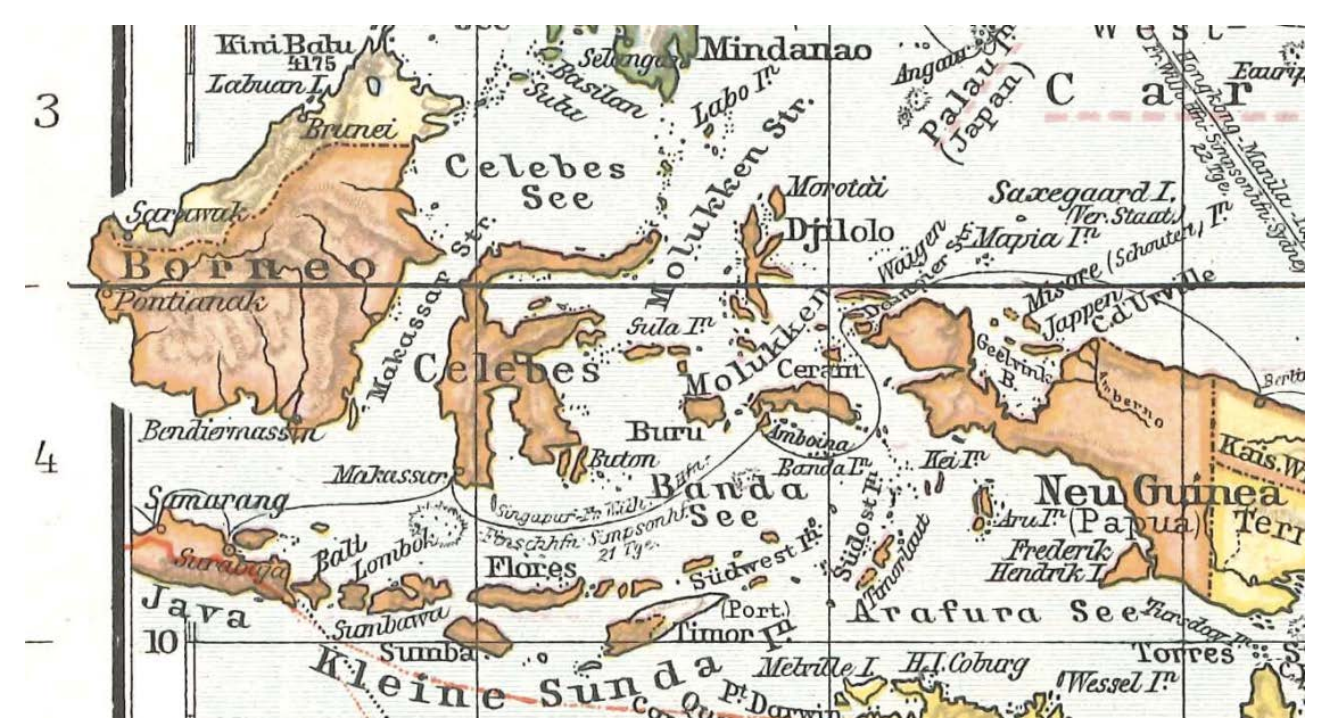

Figure 4. Copper engraving with very high toponym density.

outline of constituent polygons. We call this "touching" (see Kykladen in Figure 2 (c) ) in contrast to a full overprint such as found in Figure 2 (b).

In Table 2 we give an overview of observed strategies for group labels. In Figure 2 and 3 we provide real-world examples from a variety of cartographic sources (see references for details).

\begin{tabular}{|c|c|c|}
\hline Types & Position & Overprinting \\
\hline \multicolumn{3}{|l|}{ Straight line } \\
\hline \multirow{2}{*}{ grid aligned } & Accompany & seldom/touching \\
\hline & Through & Common \\
\hline \multirow{2}{*}{ shape aligned } & Alongside & seldom/touching \\
\hline & Through & seldom/touching \\
\hline \multicolumn{3}{|l|}{ Circular arc } \\
\hline \multirow{2}{*}{ grid aligned } & Accompany & seldom/touching \\
\hline & Through & seldom/touching \\
\hline \multirow{2}{*}{ shape aligned } & Alongside & No \\
\hline & Through & seldom/touching \\
\hline \multicolumn{3}{|l|}{ Bézier curve } \\
\hline \multirow{3}{*}{ shape aligned } & Alongside & seldom/touching \\
\hline & Through & seldom/touching \\
\hline & Connecting & No \\
\hline
\end{tabular}

Table 2. Strategies for label placement. 


\section{Algorithmic Framework}

The input to a group labeling problem is a set $S$ of $k$ features, which are given as simple polygons $P_{1}, \ldots, P_{k}$ with $n$ vertices in total. The label to be computed is a shape as well, and depending on the label type, has a baseline that is either straight, circular, or a Bézier curve. The label shape itself is the baseline offset along the perpendicular by the text height.

We first define a space of possible label designs. We include four different characteristics that may affect the labelling: label shape, point of measure, distance measure, and overlap. These characteristics are independent and, thus, our framework covers all 54 possibilities that result from the Cartesian product of these characteristics (see Table 3).

\begin{tabular}{|c|c|c|c|c|c|c|}
\hline Shape & \multirow{4}{*}{$x$} & Point of measure & \multirow{4}{*}{$\mathbf{x}$} & Distance measure & \multirow{4}{*}{$\mathbf{x}$} & Overlap \\
\hline Straight Line & & Centroid & & Min-Max & & Intersection-free \\
\hline Bezier curve & & Closest point & & Min-Sum & & Overlapping \\
\hline Circular arc & & Area & & Min-Sum-Squares & & \\
\hline
\end{tabular}

Table 3. The characteristics for labelling within our framework

The first characteristic is shape. We consider straight lines, circular arcs (being the simplest type of curves), and the commonly used cubic Bézier curves as baselines for the labels.

The second characteristic is the point of measure used to define the distance between a label and a single feature. The simplest representation of a feature is a single point, namely the centroid. The centroid, however, need not be representative of the feature. Alternatively, we can use the point of the feature that is closest to the label. If the label intersects the feature, the distance would hence be zero. Lastly, it may be the case that the label is close to the complete island, so we also use the complete area of the island to compute the distance. This definition gives rise to an integral.

Thirdly we consider the distance measure being optimized. Different distance measures are in use in cartography (Zighed 2012). We use three common ones, namely, the min-max, min-sum, and min-sum-of-squares distance measures. The min-max distance captures the largest distance between the label and the features. This measure is a measure of the 'point of worst fit'. By contrast the min-sum distance computes the sum over all distances from the label to the features. As such, the min-sum distance is a measure of average fit between shapes. A few features may be far removed from the label, however. The min-sum-of-squares distance resolves this by using the squared distance between the label and the features. The minsum-of-squares distance finds an average fit that is reasonably good at any point. 
Finally, we take the overlap with the features into account. Labels should generally not overlap their respective features. For the "inverse" problem of labeling a group of lakes (the Great Lakes, the Finger Lakes in New York State, ...), overlapping the label with the lakes may be less of a problem.

\subsection{Techniques}

We first briefly discuss some computational issues involved when determining optimal labels as well as suitable simplifications to overcome them. Then we sketch techniques which are suitable to solve the simplified problems. Algorithmic details are beyond the scope of this paper.

First, measuring distance using area is more complicated than using single points as there are an infinite number of points involved. We will simplify our problem by using a suitable finite sampling of all points of each feature, thereby discretizing the input to sets of points for every labelling setting.

Second, instead of computing a label that optimizes a measure, we will compute a center line that optimizes the measure. The center line is halfway between the baseline and the top of the label. We can extend the optimal center line to a label afterwards.

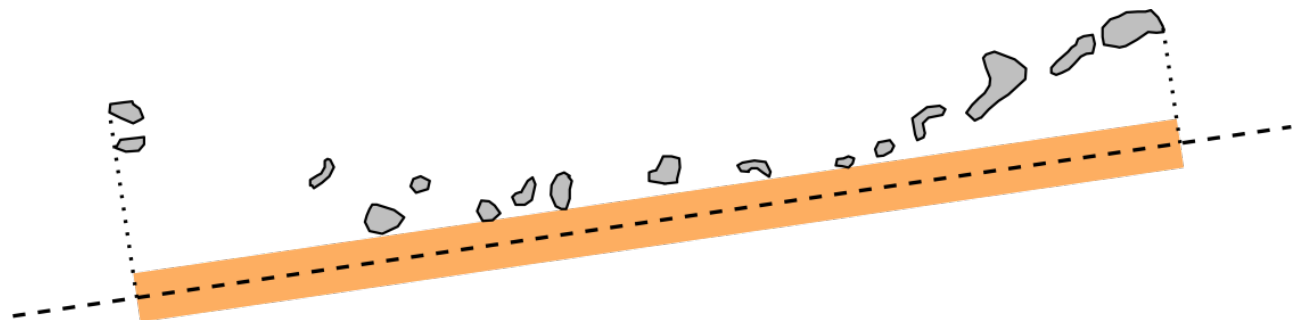

Figure 5. The label for a group of islands is assumed long enough to measure distance perpendicular to the center line for any point of the islands.

Third, we require labels that span the full extent of the feature group. We make the simplifying assumption that the width of a label can be controlled, to some degree, by changing the letter spacing. As a consequence, we consider label positions that do not need a left and a right extreme side. For straight line labels this implies that we can assume that the label length is long enough to measure all distances using perpendicular distances to the label (Figure 5). Similarly, for circular arc labels we assume that the distance from any point to the label is a perpendicular distance to a circle.

In several settings these simplifications allow us to use known algorithms from different fields for optimal labels. Moreover, computing an optimal full line or circle, rather than an optimal line segment respectively a circular arc, reduces the number of degrees of freedom in placing the label by two. Often the number of degrees of freedom has a direct influence on the effi- 
ciency of the resulting algorithm, and may show up in the some form in the exponent of the running time bound.

After computing an optimal line or an optimal circle, we can still choose the most suitable placement of the letters along the line or circle to ensure the extent is indeed fitting for the group, allowing optimality for line segments and circular arcs as well. For cubic Bézier curves we cannot use a corresponding simplification to reduce the number of degrees of freedom. Instead, we observe that we have to deal with eight degrees of freedom, two coordinates for each of the four points that defines the curve.

\section{Overlapping labels}

When we dissect the possible combinations within the framework the simplest group of settings are the "centroid-overlapping" measures. These measures do not need the fact that features are polygons. Instead we can just consider a point set for which we desire an optimal placement. Algorithms to optimize these measures over all placements are already known within the areas of computational geometry and computational statistics.

For the mix-max measure, we can compute the convex hull of the centroids and then apply a rotating calipers algorithm (Shamos 1978) to find two parallel lines that have all points in between and are closest together. The optimal line is exactly in the middle of these parallel lines. Using standard computational geometry techniques, the running time is $O(n+k \log (k))$.

For the min-sum measure, we observe that a line can only be optimal if it is a halving line: no side of the line can contain more than half of the points. Hence, the optimal line can be computed using geometric dualization (de Berg et al. 2000 - chapter 8). In dual space we can compute the median level or levels in the arrangement of lines. Using various known results (Dey 1998, Edelsbrunner \& Welzl 1986, Brodal \& Jacob 2002), we obtain an $O\left(n+k^{1.33} \log (k)\right)$ time algorithm.

The optimization of the min-sum-of-squares measure is equal to regression with the error in variables equally likely to be caused by the $x$ - and $y$-values. Deming regression, also known as the error-in-variables model, computes the optimal line minimizing the sum of squared distances in $O(n)$.

When considering the area versions of the problem the algorithms remain the same, although the number of points will be considerably higher due to the sampling of the polygons. Note that the min-max setting in combination with all-points minimizes the distance to the overall furthest point.

To measure distances to the closest points, we first replace every island by its convex hull, in $O(n)$ time (Melkman 1987), as this does not alter the result. For the min-max measure we use an algorithm that rotates two paral- 
lel lines, akin to rotating calipers, to find the optimal line in $O((n+$ $\left.\left.k^{2}\right) \log (n)\right)$ time. A variation of this algorithm solves the min-sum and minsum-of-squares versions: there are $O\left(n+k^{2}\right)$ combinatorially distinct orientations and for each, we can optimize the corresponding function.

We also mention one specific result for circles that follows immediately from known results, namely the settings where the circle can overlap the islands and minimizes the maximum distance to the centroid or to the area. This computational problem is equivalent to computing the smallest width annulus that contains a set of points; a quadratic time solution is wellknown (de Berg et al. 2000 - Chapter 7).

\section{Non-overlapping labels}

There are two different approaches to obtain algorithms for computing optimal lines in the non-overlapping settings. First, we can represent the space of all lines that do not intersect any island by using the dual plane again. The total complexity of these lines in the dual is $O\left(n+k^{2}\right)$. We can subdivide the dual space further until we can optimize a single function.

Second, we can study the geometry of the situation and derive properties that an optimal solution must have. These properties will limit the search space for the optimal label, and thus restrict the number of required computations. An example is the non-overlapping line (given our set of features) having the optimal min-sum distance to a set of points. By proving that the optimal line always passes through at least two points (of the point set or the feature points) we can develop a more efficient algorithm.

Both approaches also work to determine optimal circles. But the running time will increase and not all properties carry over from lines to circles.

\section{Comparison}

We explore the applicability of the algorithmic framework described in Section 4 by testing a subset of optimal algorithmic solutions (circular arc baselines) against digitized, manual labels. We digitized 30 circular arc labels from a variety of different Austrian, Swiss and German atlases. We then compared the labels to the automatically generated optimal labels.

We compared the manual labels with the "circular-arc * area * min-max * non-overlapping" labels resulting from our framework. Within the set of 30 labels, we found 7 instances where the resulting label was similar to the manual label. In some examples an offset of the island group was required for the label to match the manual label. Figure 5 (a) shows an example including both the manual and computed label for the Ryukyu islands. 


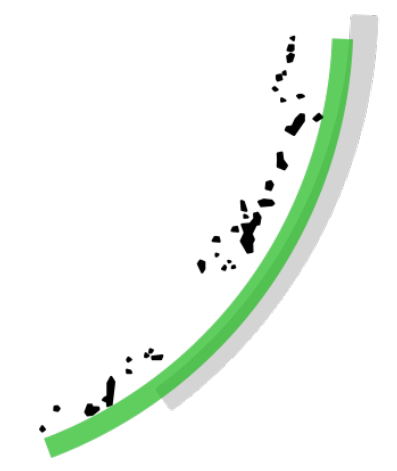

(a)

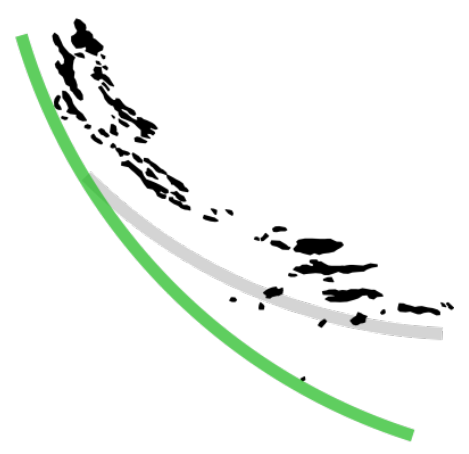

(b)

Figure 5. Manual (grey) and automated (green) labels for different island groups. (a) The Ryukyu islands. The automatically generated label matches reasonably well, but requires an offset from the group. (b) The Dalmatian islands. Two problems interfere with automated labelling. The min-max measure focusses only on the point of worst fit and the label can be only on the outside of the group of features.

While several manual labels fit the min-max distance measure well, our exploration indicates that the min-max measure is not the main criterion applied by cartographers. It may lead to situations where the descriptive function of the label is sacrificed for the benefit of a single outlier of the group (Figure 5 (b)). Using an alternative geometric measure may solve this problem. For example, the min-sum-of-squares measure takes the average distance of all features into account. Unfortunately, we do not yet have optimal algorithms for min-sum-of-squares circular arc labels and, hence, cannot test this hypothesis. A better circular arc label would also be possible if we do not require the label to be on the outside of the group of islands. Unfortunately, we do not yet know how to find such labels optimally. We believe in "objectivity through optimality" and hence will test these settings once we or others succeeded in developing optimal algorithms.

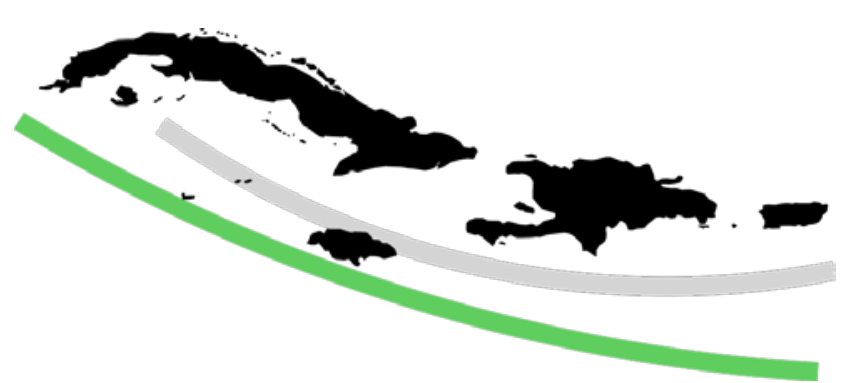

Figure 6: Antilles. The manual label (grey) completes the group and is less likely to be captured by a geometric distance measure.
Our current framework takes into account the distance to features only, but does not yet consider the shape and extent of the whitespace around the group. As already mentioned by Wood (2000), labels may either follow the general shape of the group or complete its 
overall shape. The second option is mainly tied to the whitespace around the feature group (Figure 6). The label does not necessarily optimize the geometric distance to the group of features, but completes the geometric shape of the group. Labels may also be used to balance the visual weight of the feature group.

During our comparisons, it became apparent that several label positions could be reproduced with the framework but with an inverted curvature. The manual samples nearly always prefer to tie the features together like a pincer or enclose them like a bracket. This is in contrast with the actual curvature of the boundary of the group which might bend away (Figure 7 (a)).
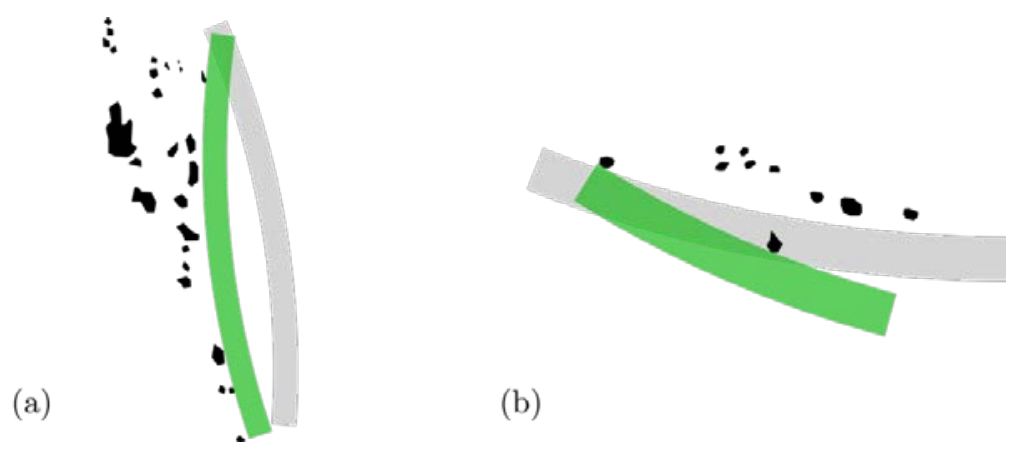

(b)

Figure 7. (a) New Hebrides. Manual labels (grey) are often concave towards the island groups, the automated label has a lower distance to the islands. (b) Society Islands. Requiring non-overlapping labels may be overly restrictive.

As expected, small overlaps with the features are frequently used in manually placed labels and hence such an option should be included in our framework.

\section{Conclusion}

We outlined an approach to quantitatively study the labelling of feature groups by comparing manually generated labels to a set of formally defined, algorithmically optimal placements. In the absence of cartographic guidelines for this important labeling problem, we believe that our systematic study will eventually lead to a set of clear specifications which are suitable for automated construction of high-quality labels that match best manual practices. First results are encouraging, but clearly extensive future work is needed to develop optimal algorithms and similarity measures, extend the framework, and hopefully finally validate our approach.

Acknowledgements. Thanks to Sarah Lohr and Susanne Heuser for the digitizing maps. 


\section{References}

Barrault M (2001) A Methodology for Placement and Evaluation of Area Map Labels. Computers, Environment and Urban Systems 25(1): 33-52

Barrault M, Lecordix F (1995) An Automated System for Linear Feature Name Placement which Complies with Cartographic Quality Criteria. Proc. AutoCarto 12: 321-330. ACSM/ASPRS

De Berg M, Cheong O, Van Kreveld M, Overmars M (2008) Computational Geometry, 3rd edition. Springer

Bertin J (1967) Semiology of Graphics: Diagrams, Networks, Maps. Univ. of Wisconsin Press, 1983

Brewer C (2005) Designing Better Maps: A Guide for GIS Users. ESRI Press

Brodal G, Jacob R (2002) Dynamic planar convex hull. Proc. 43rd Annual IEEE Symp. on Foundations of Computer Science: 617-626, IEEE

Chirié F (2000) Automated Name Placement With High Cartographic Quality: City Street Maps. CaGIS 27(2): 101-110

Christensen J, Marks J, Shieber S (1995) An Empirical Study of Algorithms for Point-Feature Label Placement. ACM Trans. on Graphics 14(3): 203-232

Dey T (1998) Improved Bounds for Planar k-Sets and Related Problems. Discrete Comput. Geom. 19 (3): 373-382

Edmondson S, Christensen J , Marks J and Shieber S (1996) A General Cartographic Labeling Algorithm. Cartographica 33(4): 13-23

Edelsbrunner H, Welzl E (1986) Constructing belts in two-dimensional arrangements with applications. SIAMJ . on Computing, 15(1), 271-284

Imhof E (1962) Die Anordnung der Namen in der Karte. Int. Yearb. of Cartography, 93-129, Kirschbaum

Kern J , Brewer C (2008) Automation and the Map Label Placement Problem: A Comparison of Two GIS Implementations of Label Placement. Cartographic Perspectives $60,22-45$

Melkman A, (1987) On-line construction of the convex hull of a simple polyline. Information Processing Letters 25(1): 11-12

Lloyd R (1997) Visual Search Processes Used in Map Reading. Cartographica 34(1): 11-32

Noyes L (1980) The Positioning of Type on Maps: The Effect of Surrounding Material on Word Recognition Time. Human Factors 22(3): 353-360

Phillips R, Noyes L, Audley R (1977) The Legibility of Type on Maps. Ergonomics (6): 671-682

Rylov M, Reimer A (2014a) A Comprehensive Multi-Criteria Model for High Cartographic Quality Point-Feature Label Placement. Cartographica 49(1): 52-68 
Rylov M, Reimer A (2014b) A Practical Algorithm for the External Annotation of Area Features. Carto.J .

Shamos M (1978) Computational geometry. Ph.D. thesis. Dept. of CS, Yale Univ.

Steiniger S, Weibel R (2007) Relations among Map Objects in Cartographic Generalization. CaGIS 34(3): 175-197

Van Dijk S, Van Kreveld M, Strijk T, Wolff A (2002) Towards an Evaluation of Quality for Names Placement Methods. Int. J . of GIS 16(7): 641-661

Van Kreveld M, Schlechter T (2005). Automated label placement for groups of islands. Proc. 22th Int. Cart. Conf.

Van Kreveld M, Strijk T, Wolff A (1999) Point Labeling with Sliding Labels. Comp. Geom.: Theory \&Appl. 13: 21-47

Van Roessel W (1989) An Algorithm for Locating Candidate Labeling Boxes Within a Polygon. American Cartographer 16(3): 201-209

Wolff A, Strijk T (2009) A map labeling bibliography. Available at http://i1lwww.iti.uni-karlsruhe.de/ awolff/map-labeling/ bibliography/

Wood C (2000) Descriptive and Illustrated Guide for Type Placement on Small Scale Maps. Carto. J . 37(1): 5-18

Yoeli P (1972) The Logic of Automated Map Lettering. Carto. J . 9(2): 99-108

Zighed J , Abdesselam R, Hadgu A (2012) Topological Comparisons of Proximity Measures. Advances in Knowledge Discovery and Data Mining: 379-391

\section{List of Maps}

Fig. 1) Bertalan (2006): Cabo Verde 1:200 000, 6th Ed. (verso 1:750 000), AB Kartenverlag.

Fig. 2a) Apenninen- und Südosteuropäische Halbinsel, 1:5 000 000. In: Schandl, H. (Ed.) (1982) Ed. Hölzel Österreichischer Oberstufen-Atlas, p. 45.

Fig. 2b) Australien und Neuseeland, 1:30 000 000. In: Imhof, E. (1976) Schweizerischer Mittelschulatlas, 17th Ed., p. 128, Orell Füssli.

Fig. 2c) Östliche Mittelmeerländer (1978), 1:5 000 000. In: Große, G and Kötter, H. (Ed.) List Großer Weltatlas - Mensch und Erde, p. 88, List Verlag.

Fig. 3a) International Map of the World (Aviation edition) 1:1000 000, N-G-38, Sheet Er-Riyad, Sonderausgabe Nr. 2 Stand VI.1942, German General Staff.

Fig. 3b) International Map of the World 1:1000 000, Sheet N-G-37 Medina, Sonderausgabe Nr. 2 Stand VI.1942, German General Staff.

Fig. 3c) Croquis de L'Afrique au 1:1000 000 (Aviation edition), N-G-34, 1939, French Army Geographical Service.

Fig. 3d) Britische Inseln und Nordsee, 1:4 000 000. In: Imhof, E. (1976) Schweizerischer Mittelschulatlas, 17th Ed., p.66, Orell Füssli.

Fig. 4) Australien und Polynesien, 1:50 000 000. In: Gaebler, E (1926) Eduard Gaeblers Hand-Atlas über alle Teile der Erde, 13th Ed., p. 32. 